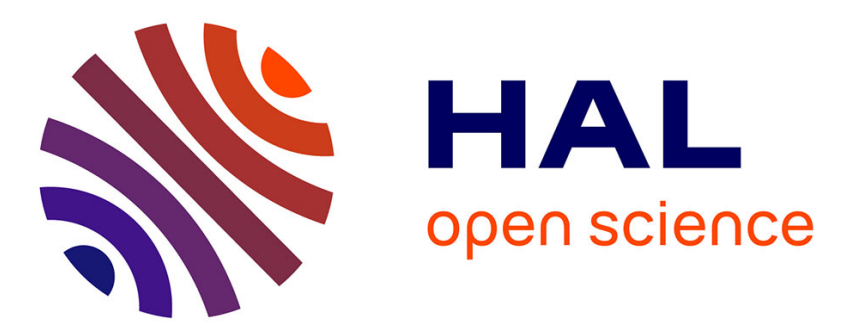

\title{
Influence of the geometry of protective barriers on the propagation of shock waves
}

\author{
Isabelle Sochet, Sébastien Eveillard, Jean-Yves Vinçont, P.-F. Piserchia, \\ Xavier Rocourt
}

\section{- To cite this version:}

Isabelle Sochet, Sébastien Eveillard, Jean-Yves Vinçont, P.-F. Piserchia, Xavier Rocourt. Influence of the geometry of protective barriers on the propagation of shock waves. Shock Waves, 2016, 27 (2), pp.209 - 219. 10.1007/s00193-016-0625-4 . hal-01389895

\section{HAL Id: hal-01389895 \\ https://hal.science/hal-01389895}

Submitted on 30 Oct 2016

HAL is a multi-disciplinary open access archive for the deposit and dissemination of scientific research documents, whether they are published or not. The documents may come from teaching and research institutions in France or abroad, or from public or private research centers.
L'archive ouverte pluridisciplinaire HAL, est destinée au dépôt et à la diffusion de documents scientifiques de niveau recherche, publiés ou non, émanant des établissements d'enseignement et de recherche français ou étrangers, des laboratoires publics ou privés. 


\title{
Influence of the geometry of protective barriers on the propagation of shock waves
}

\author{
I. Sochet ${ }^{1}$ - S. Eveillard ${ }^{2}$ - J. Y. Vinçont ${ }^{2}$ - P. F. Piserchia ${ }^{2}$ - X. Rocourt ${ }^{1}$
}

Received: 6 July 2015 / Revised: 30 January 2016 / Accepted: 4 February 2016

(C) Springer-Verlag Berlin Heidelberg 2016

1 Abstract The protection of industrial facilities, classified 2 as hazardous, against accidental or intentional explosions 3 represents a major challenge for the prevention of personal 4 injury and property damage, which also involves social and economic issues. We consider here the use of physical barriers against the effects of these explosions, which include the pressure wave, the projection of fragments and the thermal flash. This approach can be recommended for the control of major industrial risks, but no specific instructions are available for its implementation. The influence of a protective barrier against a detonation-type explosion is studied in small-scale experiments. The effects of overpressure are examined over the entire path of the shock wave across the barrier and in the downstream zone to be protected. Two series of barrier structures are studied. The first series (A) of experiments investigates two types of barrier geometry with dimensions based on NATO recommendations. These recommendations stipulate that the barrier should be $2 \mathrm{~m}$ higher than the charge height, the thickness at the crest should be more than $0.5 \mathrm{~m}$, while its length should be equal to twice the protected structure length and the bank slope should be equivalent to the angle of repose of the soil. The second series (B) of experiments investigates the influence of geometrical parameters of the barrier (thickness at the crest and inclination angles of the front and rear faces) on its protective effects. This project leads to an advance in our understanding of the physical phenomena involved in

Communicated by C.-Y. Wen.

I. Sochet

isabelle.sochet@insa-cvl.fr

1 INSA Centre Val de Loire, Université d'Orléans, PRISME EA 4229, Bourges, France

2 CEA DAM, DIF, 91297 Arpajon, France the propagation of blast waves resulting from an external explosion, in the area around a protective physical barrier. The study focuses on the dimensioning of protective barriers against overpressure effects arising from detonation and shows the advantage of using a barrier with a vertical front or rear face.

Keywords Protective barrier - Explosion effect - Shock wave $\cdot$ Blast load $\cdot$ Detonation

28 29 30 31 32 33

\section{Introduction}

At industrial sites, whether public or private, one of the major concerns in modern society is the safety of goods and people with respect to the risks associated with explosions of either accidental or malicious origin. The disasters at AZF in 2001 (Toulouse, France), at the Nitrochimie dynamite factory in 2003 (Billy-Berclau, France), at a fireworks storage facility at Kolding in 2004 (Denmark) and at the West Fertilizer Company plant in 2013 (Texas, USA) are examples showing that "zero risk does not exist". To limit the occurrence of new accidents, companies have a panoply of safety measures involving prevention or protection against the risks inherent in any accident.

The detonation of an explosive charge causes mechanical effects, such as overpressure, heating and possible effects related to the projection of fragments. The presence of a protection barrier (walls, fill and slope) ensures the easy protection of installations and people against the heating effects of an explosion and the projection of fragments. However, protection from the effects of overpressure is not guaranteed simply by the presence of a physical protection barrier of unspecified form. Indeed, the interaction of a shock wave
36

37 38 39 40 41 42 43 44 45 46 47 48 
with a structure is difficult to predict and depends on many parameters.

To our knowledge, few studies have been carried out on the optimization of protective barriers. With this objective in mind, Zhou and Hao [1] used digital simulations to study the effectiveness of a protective wall placed in front of a building. Their study demonstrated that the reduction of blast load does not depend solely on the height of the protective wall, the distance between the centre of the explosion and the barrier, the distance between the barrier and the building or the height of the building. The effect of wall thickness was studied but did not contribute significantly to the blast load behind the wall.

The medium-scale experimental study carried out by Allain [2] comprised barriers with two inclined slopes of $45^{\circ}$ without a flat crest and using a height of $1.5 \mathrm{~m}$. The tests were conducted using spherical charges of TNT ( 8 and $37 \mathrm{~kg}$ ) and composition B $(50 \mathrm{~kg})$. The distance between the charge and the obstacle $(d)$ varied from 0.75 to $17 \mathrm{~m} / \mathrm{kg}^{1 / 3}$. These medium-scale tests demonstrated that a barrier, according to its geometry and form factors, can lead to various flow modes. The protective barrier considered in this case accentuated the positive overpressure of the shock wave and thus did not show a protective effect. These results have been confirmed by the simulations of Borgers [3], who noted that the relaxation on the rear face of a Mach stem results from reflection on the front face or from an incident wave (for a regular reflection) for certain configurations. This can lead to an accentuation of the reflection of the shock wave on the ground downstream of the obstacle (according to the nature of the wave and the angle of inclination of the wall).

Thus, the recommendations of NATO evoked in the "Guide to good practices in pyrotechnics" [4] estimate a minimal thickness of $0.5 \mathrm{~m}(e>0.5 \mathrm{~m})$ and specify that the height of the barrier must be more than $2 \mathrm{~m}$ higher than the highest point of the charge.

Therefore, the objective of the present study was to evaluate the protective effects related to the overpressure of barriers according to their form and size. The barriers represent protective obstacles placed in the path of the shock wave that are intended to mitigate its effects (such as overpressure and impulses). The obstacle is assumed to be infinitely rigid, so the reflection is considered as "perfect" over all its surface.

In this study, we consider a generic barrier typology (Fig. 1) with the following preset parameters: mass charge $(W)$, height and width at crest of the obstacle $(H, e)$, slope angles of the barrier faces with respect to the ground $(\alpha)$, distance between charge and barrier $(d)$. In this study, the explosive charge is placed only at ground level.

Small-scale experiments are carried out using three types of barrier model. Small-scale tests have many advantages. Indeed, their cost is low and accurate laboratory methods can be applied. Moreover, the test conditions are well controlled

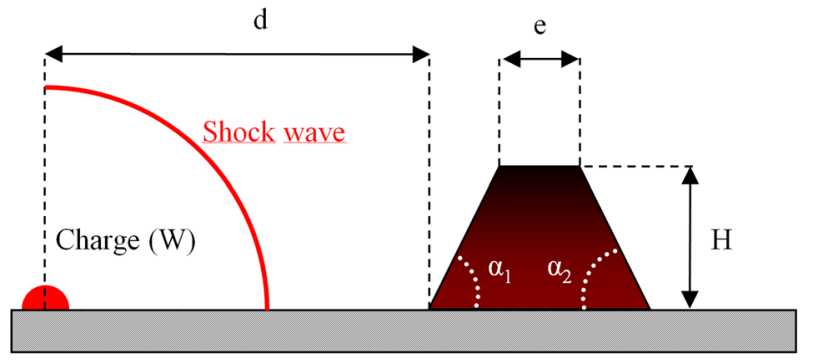

Fig. 1 Schematic diagram of a protection barrier- $W$ charge mass $(\mathrm{kg}$ of TNT), $d$ distance between centre of charge and the front face (m), $e$ thickness at crest of obstacle (m), $H$ height of barrier (m), $\alpha_{1}$ angle of inclination of front face $\left(^{\circ}\right), \alpha_{2}$ angle of inclination of rear face $\left(^{\circ}\right.$ )

and independent of the weather, the reproducibility of test conditions can be readily ensured, and it is easy to establish parametric studies and vary the geometric dimensions of the studied structures.

\section{Experimental set up}

\subsection{Experimental details}

The experiments are conducted at a small scale on a test bench [5-7].

The explosive charges are made up of a stoichiometric mixture of propane and oxygen gases. The hemispherical charges used are positioned on the ground, initially confined in a soap bubble. Two charge radii are used: $R_{1}=0.06 \mathrm{~m}$ and $R_{2}=0.03 \mathrm{~m}$. The explosive charge is initiated by an exploding wire [6]. In the analysis of results (next section), the charge radius will be noted by $\mathrm{R} 0$ with its specified value.

The zone of experimentation (Fig. 2) is divided into two sectors relative to the centre of the explosive charge [5]: the free field zone and the zone of investigation. In the free field zone, the incident shock wave resulting from detonation of the explosive charge propagates without interaction with the structure. The pressure sensors placed in this zone, called "reference sensors", are used to check the reproducibility of the detonations. The structure is placed in the zone of investigation along with the explosive charge. Within this zone of investigation, an additional zone can be identified in which the protection barrier prevents arrival of the wave.

The dimensions and positioning of the protection barrier depend on the studied configuration (series 1 and 2). Pressure sensors (piezoelectric, PCB) are placed flush with the structure to detect possible couplings of the various physical phenomena (reflection, relaxation and recombination of shock waves) as well as downstream from the barrier to study the protective effect. Each position is identified by a distance in direct line with the charge, which is defined by the distance between the position of the sensor and the centre of the explosive charge. 
Fig. 2 Schematic diagram of the experimental setup

\subsection{Tested configurations}

The geometrical configurations and dimensions of the barriers studied in small-scale experiments are designed to analyse several physical phenomena (reflection, relaxation and recombination of shock waves) as well as the protective effect of barriers according to their geometry. The test campaigns included two series of barriers and a configurationfree field (without obstacles), allowing characterization of the evolution of various mechanical wave parameters for a gas load as a function of the distance travelled by the wave. The first series of barriers (A: 1A, 2A) is designed to study two barrier geometries based on the recommendations of NATO [4]. The second series (B: $1 \mathrm{~B}, 2 \mathrm{~B})$ is designed to study the influence of two geometrical parameters of the barrier (thickness at crest and slope angles of the front and rear faces) on the protective effect of the barrier. In this study, the impact of bypassing waves is not analysed, which means that, for these two experimental series, we assume a protective barrier of infinite length.

\subsubsection{Configurations with barriers—series A}

The first series of protective barrier geometries is designed based on the recommendations of NATO for two gas loads ( $R_{1}$ and $R_{2}$ ) and using the Hopkinson scale [8] for a scaling factor $k(k=15)$. The protective barriers, $1 \mathrm{~A}$ and $2 \mathrm{~A}$, are dimensioned according to the recommendations for the two tested gas loads.

The charge radius $\left(R_{1}\right)$ is $0.06 \mathrm{~m}$, and its detonation releases an energy of $13.75 \times 10^{-3} \mathrm{MJ}\left(E_{k}\right.$ on scale $\left.1 / k\right)$. Thus, for example, for a dimensional scaling factor $k$ of 15 , the released energy on the real scale is $46.41 \mathrm{MJ}$ ( $E_{1}$ on a scale of 1/1).

The distance between the centre of the charge and the obstacle varies between 0.07 and $0.10 \mathrm{~m}$ (or between 1.05 and $1.50 \mathrm{~m}$ on the real scale). The dimensions of the first protective barrier (1A) follows the recommendations of NATO for a gas load of radius $R_{1}$. The height of the barrier can be calculated from the following equation (1):

$H_{1 / k}=\frac{2}{k}+$ Radius or $H_{1 / k} \approx 0.19 \mathrm{~m}$ and $H_{1 / 1}=2.85 \mathrm{~m}$, charge radius $\left(R_{1}\right)$.

The thickness at the crest of the protection barrier can be estimated from the following equation (2):

$e_{\min , 1 / k}=\frac{0.5}{k}$ or $e_{\min , 1 / k} \approx 0.03 \mathrm{~m}$

The minimal width of the protective barrier at the top of the explosive charge can be evaluated from the following equation (3):

$e_{\text {charge }, 1 / k}=\frac{0.9}{k}$ or $e_{\text {charge }, 1 / k} \approx 0.06 \mathrm{~m}$.

The first protective barrier (1A) is dimensioned based on the recommendations for a gas blast load of radius $R_{1}$ (Fig. 3).

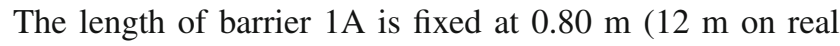
scale).

The second protective barrier (2A) is dimensioned based on the height recommended by NATO for a gas load of radius $R_{2}=0.03 \mathrm{~m}$. To allow a proper comparison of the two geometries, the wave path length (i.e., distance travelled by the shock wave) over barrier 2B must be almost identical or close to that for barrier 1A (Fig. 4). The height is thus estimated at $0.16 \mathrm{~m}$, and the thickness is fixed at $0.06 \mathrm{~m}$ (distances travelled over the trapezoidal profile are $0.527 \mathrm{~m}$ for barrier $1 \mathrm{~A}$ and $0.513 \mathrm{~m}$ for barrier $2 \mathrm{~A}$, with a difference of $3 \%$ ). This second geometry allows us to analyse the influence of barrier height and thickness on the protective effect for two explosive loads $\left(R_{1}\right.$ and $\left.R_{2}\right)$. Barriers $1 \mathrm{~A}$ and $2 \mathrm{~A}$ have the same length: $L=0.80 \mathrm{~m}$.

The distances travelled by the wave passing over the top crest of the barriers 1A (Fig. 3) and 2A (Fig. 4) are very close, 0.527 and $0.513 \mathrm{~m}$, respectively. 


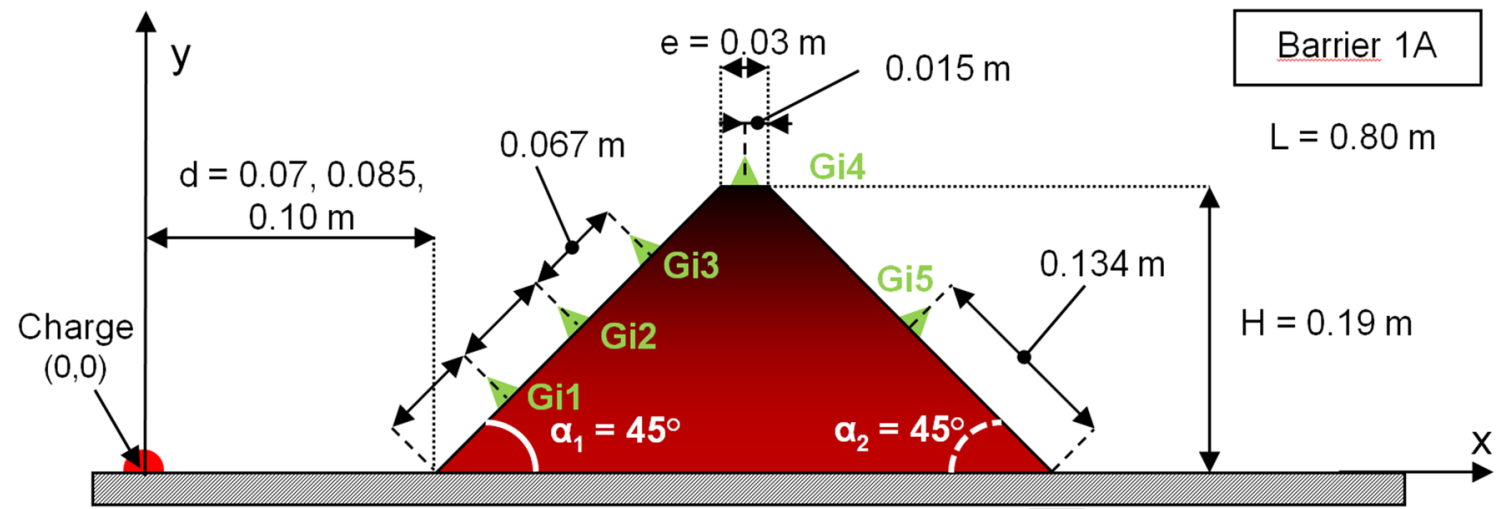

Fig. 3 Dimensions of barrier 1A with gauge positions-view in the plane $z=0$

Fig. 4 Dimensions of barrier $2 \mathrm{~A}$ with positions of sensors-view in the plane $z=0$

Fig. 5 Dimensions of barrier $1 \mathrm{~B}$ with positions of sensors-view in the plane $z=0$
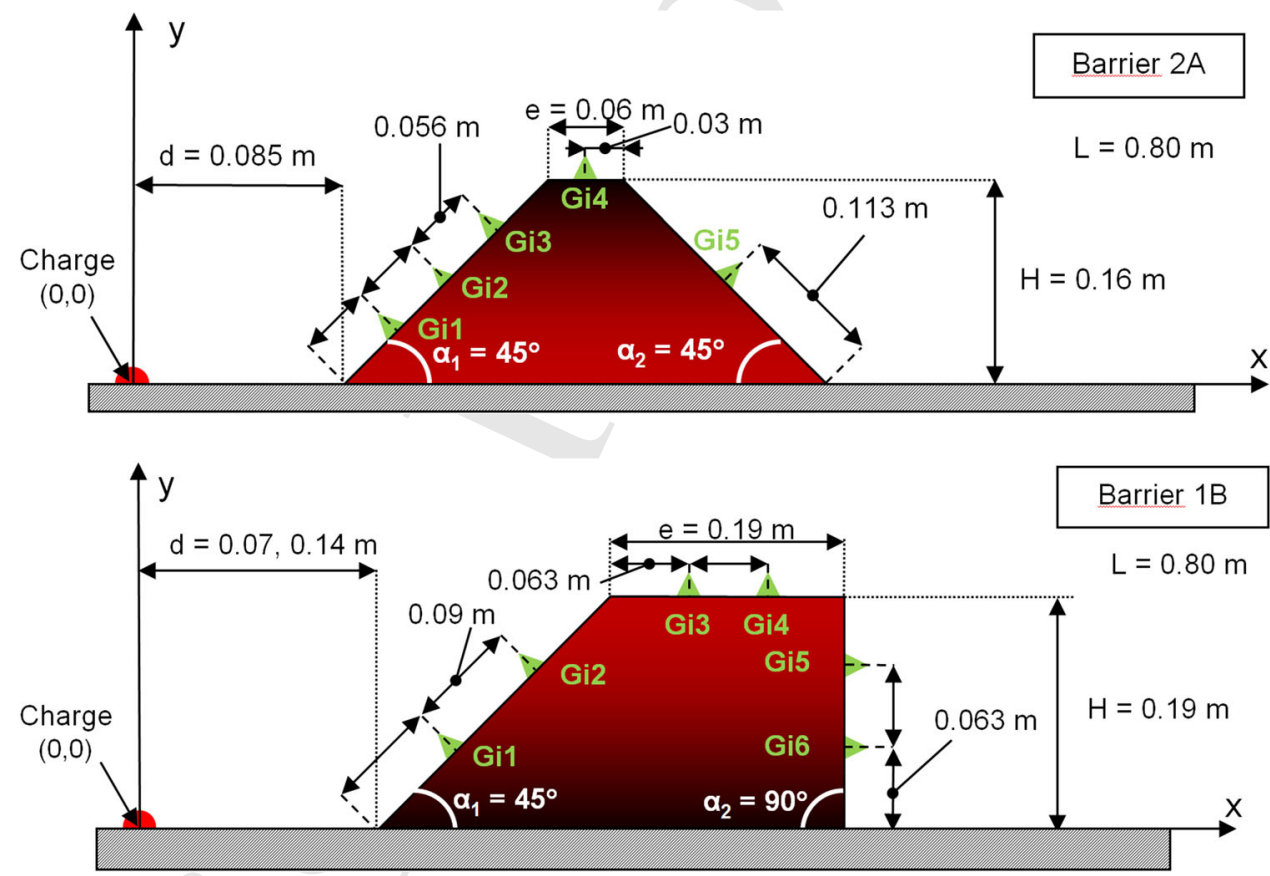

\subsubsection{Configurations with barriers—series B}

The objective of series B is to study the influence of the wall inclination angle (upstream and downstream of the barrier) on the physical phenomena occurring during interaction of the shock wave with a protective barrier and to analyse its impact on the protective effect. The slopes of the front and rear faces are set at two angles: $45^{\circ}$ and $90^{\circ}$. The thickness across the crest of the protective barrier is equal to its height to allow decoupling of the physical phenomena $(e=H=$ $0.19,2.85 \mathrm{~m}$ on real scale, for $k=15$ ) and thus create an attenuating wave with greater amplitude than for a barrier dimension based on the minimal thickness recommended by $\operatorname{NATO}\left(e_{\min }=0.5 \mathrm{~m}\right)$.

Barrier 1B has a front face at $45^{\circ}$ and a rear face at $90^{\circ}$ (Fig. 5). The distance of the path of the shock wave over the barriers $1 \mathrm{~B}$ and $2 \mathrm{~B}$ is fixed at $0.80 \mathrm{~m}$.
Barrier 2B is built with an inclination angle of $90^{\circ}$ for the front face and $45^{\circ}$ for the rear face (Fig. 6).

Barriers 1B and 2B have identical sizes, with equal distances covered by the wave passing across the top face $(0.649 \mathrm{~m})$. The distances of sensors are summarized in Fig. 7 .

\section{Analysis of phenomena on barrier A}

We first examine the variation of overpressure as a function of the scaled distance of the path of the shock wave over the barrier (Fig. 8). The reduced distance $Z$ is defined by

$Z=\frac{R}{\sqrt[3]{\mathrm{m}}}, \quad Z=\left[\mathrm{m} \mathrm{kg}^{-1 / 3}\right]$,

where $R[\mathrm{~m}]$ represents the standoff distance from the centre of the explosive charge to the point of interest and $m$ is the 


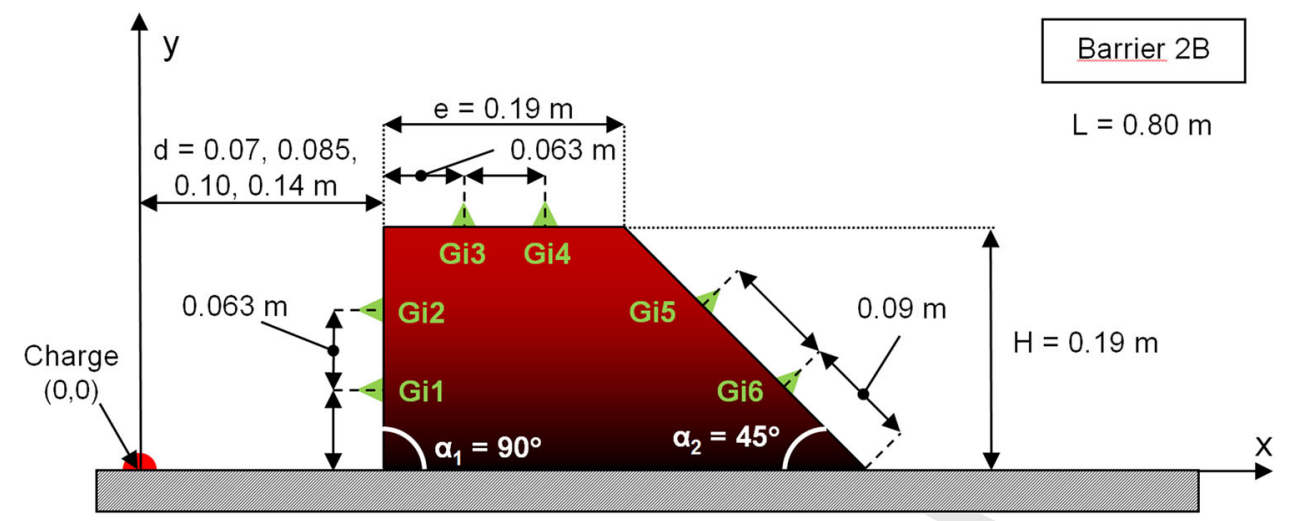

Fig. 6 Dimensions of barrier 2B with positions of sensors-view in the plane $z=0$

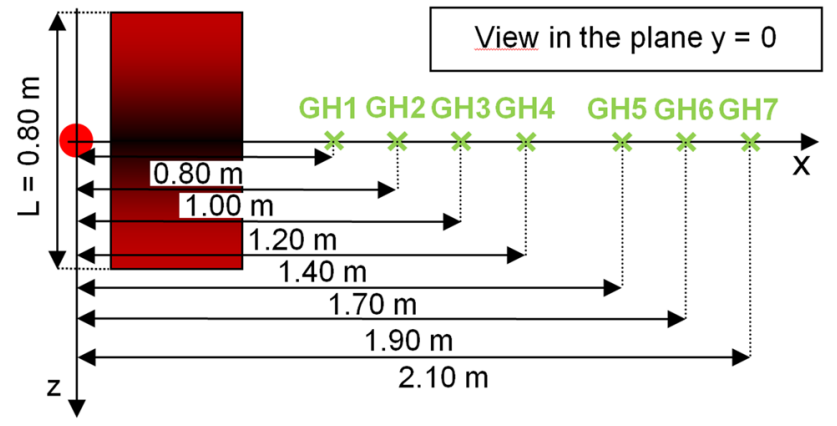

Fig. 7 View in plane $y=0$ showing barriers $\mathrm{A}$ and $\mathrm{B}$ with positions of sensors behind the barrier mass of gas load in $\mathrm{kg}$ based on a spherical charge of radius R0.

\subsection{Reflection on the front face of barriers $1 \mathrm{~A}$ and $2 \mathrm{~A}$}

The distance between the centre of the explosive charge and the point of interest allows us to obtain the incident Mach number by using the maximum incident overpressure $\left(\Delta P^{+}\right)$ calculated from empirical formulas.

The evolution of the overpressure of a blast wave $\left(\Delta P^{+}\right)$ can be estimated for a TNT charge based on the formula of Kinney [8] in equation (5) $\left(\Delta P^{+}\right.$in $\mathrm{Pa}$ with $P_{0}=$ $101,325 \mathrm{~Pa}, W$ mass in $\mathrm{kg}$ of TNT, $Z$ in $\mathrm{m} \mathrm{kg}^{-1 / 3}$ )

$$
\frac{\Delta P^{+}}{P_{0}}=\frac{808 \times\left[1+\left(\frac{Z}{4.5}\right)^{2}\right]}{\sqrt{1+\left(\frac{Z}{0.048}\right)^{2}} \times \sqrt{1+\left(\frac{Z}{0.32}\right)^{2}} \times \sqrt{1+\left(\frac{Z}{1.35}\right)^{2}}}
$$

For a given gas load (stoichiometric propane-oxygen combustion), the evolution of the overpressure of a blast wave $\left(\Delta P^{+}\right)$can be estimated from equations (5) and (6) for an energy-scaled distance to the point of combustion of a spherical gas load $\left(\lambda\right.$ in $\left.\mathrm{m} / \mathrm{MJ}^{1 / 3}\right)[5]$ :

$\ln \left(\frac{\Delta P^{+}}{P_{0}}\right)=0.0895-1.7633 \times \ln (\lambda)+0.1528 \times \ln (\lambda)^{2} \quad 259$

$$
-0.0066 \times \ln (\lambda)^{3}-0.0021 \times \ln (\lambda)^{4},
$$

or according to the distance scaled with respect to the cubic root of the mass of the explosive gas load $\left(Z\right.$ in $\left.\mathrm{m} \mathrm{kg}^{-1 / 3}\right)$ [6]:

$$
\begin{aligned}
\ln \left(\frac{\Delta P^{+}}{P_{0}}\right)= & 1.486-1.782 \times \ln (Z)-0.104 \times(\ln (\mathrm{Z})) \\
& +0.115 \times(\ln (Z))^{3}-0.017 \times(\ln (Z))^{4}
\end{aligned}
$$

Hence, Fig. 8 shows clearly that all overpressure values on the front face are higher than the overpressures corresponding to the free field. The divergent spherical incident wave is reflected on the front face of the protective barrier. The incident Mach number $\left(M_{1}\right)$ of the shock wave can be obtained from the maximum of incident overpressure $\left(\Delta P^{+}\right)$and the initial pressure $\left(P_{0}=101,325 \mathrm{~Pa}\right)$ and $\gamma=1.4$ by using equation (7):

$M_{1}=\sqrt{\frac{1}{2 \times \gamma} \times\left((\gamma+1) \times \frac{P_{0}+\Delta P^{+}}{P_{0}}+(\gamma-1)\right)}$.

The reflection mode (regular reflection or Mach reflection) can vary according to the position and dimensions of the bar$\operatorname{rier}\left(d, H, \alpha_{1}\right)$. The point of transition between these reflection modes can be determined by the simplified relation (8) due to Kinney [8], expressed as a function of the Mach number of the incident wave $\left(M_{1}\right)$ :

$\beta_{1 \lim }=\frac{1.75}{M_{1}-1}+39$. 
Fig. 8 Overpressure as a function of scaled distance on barrier A

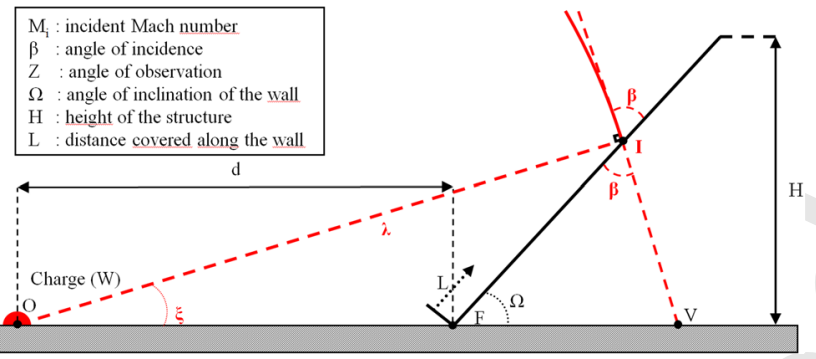

Fig. 9 Schematic diagram of incident shock wave at impact point I

This angle of transition is compared to the angle of incidence calculated from the geometrical relations derived from Fig. 9.

Equations (10) and (11) allow us to determine the angle of incidence $(\beta)$ and the angle of observation $(\zeta)$.

$\zeta=\arctan \left(\frac{\sin \alpha \times L}{\cos \alpha \times L+d}\right)$ with $L \in\left[0 ; \frac{\mathrm{H}}{\sin \alpha}\right]$

$\beta=\frac{\pi}{2}-\alpha+\xi$

An analysis of the overpressures obtained on barriers 1A and 2A leads us to estimate the formation of a Mach stem on the face before the barrier. This observation of reflection modes is also confirmed using the curves presented in TM51300 for the overpressures considered here [9].

\subsection{Relaxation on the front face of barriers $1 \mathrm{~A}$ and $2 \mathrm{~A}$}

The reflection mode (regular reflection or Mach reflection) at the impact point on the front face of the protective barrier defines the nature of the wave that is propagated downstream from the barrier (incident wave or Mach stem). During the passage of this wave between the impact point and the top of the protective barrier, the wave undergoes a relaxation phenomenon.

This physical phenomenon leads to an attenuation of the maximum overpressure in the field close to the edge between the front face and the top of the protective barrier for $Z=3-3.3 \mathrm{~m} \mathrm{~kg}^{-1 / 3}$ for $R_{2}$ loads $(0.06 \mathrm{~m})$ and $Z=5.9-6.4 \mathrm{~m} \mathrm{~kg}^{-1 / 3}$ for $R_{1}$ loads $(0.03 \mathrm{~m})$ (Fig. 8). The maximum overpressure values are less than the free field values, thus contributing to the appearance of a protective effect downstream from the barrier. During wave propagation on the top part of the barrier, maximum overpressure is attenuated by the distance covered by the wave (network of relaxation waves downstream from the shock front).

The variation in maximum overpressure between the three configurations arises from the intensity of the incident wave at the top of the barrier.

In this zone of interest, the difference between the two obstacles (barriers 1A and 2A) corresponds to the thickness of the barrier (greater thickness for barrier $2 \mathrm{~A}, e_{2 \mathrm{~A}}=e_{1 \mathrm{~A}} \times 2$, the attenuation effect per distance covered is thus slightly more marked), as shown in Fig. 8.

\subsection{Relaxation on the downstream face of barriers 1A and $2 \mathrm{~A}$}

During the passage of the shock wave between the top and the rear face downstream of the barrier, the wave is subject to a second relaxation. 
Fig. 10 Overpressure as a function of scaled distance on barrier B

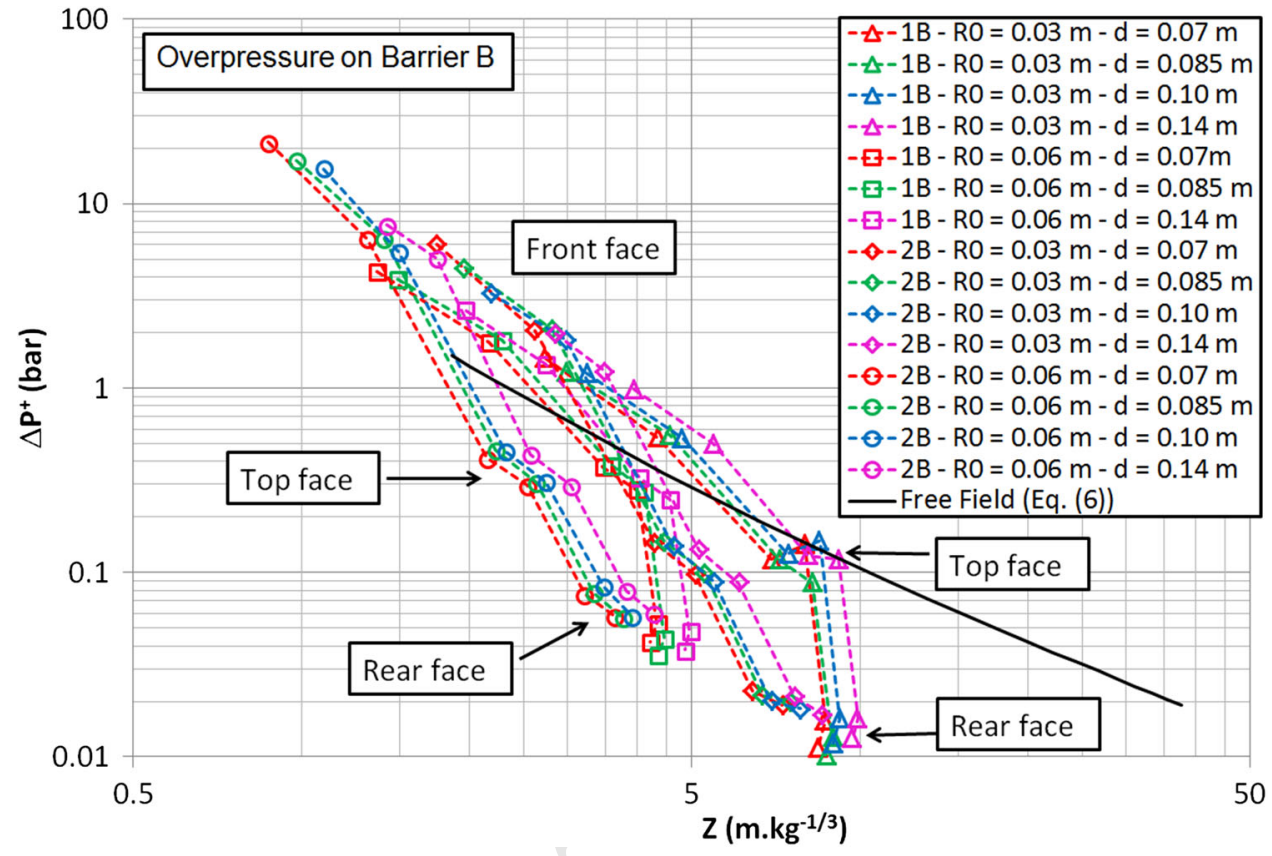

This phenomenon leads to an attenuation of the maximum overpressure across the rear face of barriers $1 \mathrm{~A}$ and $2 \mathrm{~A}$ (Fig. 8).

The maximum overpressures in the free field are higher than the values on the face downstream of the barrier $\left(\Delta P_{\text {free field }}^{+} \gg \Delta P_{\text {barrier }}^{+}\right.$).

\section{Analysis of phenomena on barrier B}

We now examine the variation of overpressure as a function of the scaled distance $Z$ defined by the relation (4) on the path of the shock wave over the barrier (Fig. 10).

\subsection{Reflection on barrier 2B}

Barrier 2B has the same downstream face as barrier 1A. The physical phenomena on this barrier surface are thus of comparable nature, i.e., reflection of the incident wave on a plane inclined at $45^{\circ}$ and appearance of a Mach stem. These two geometries are only different at the top of the protective barrier, with a much greater thickness at the top of barrier 2B $\left(e_{2 \mathrm{~B}}=0.19 \mathrm{~m} \gg e_{1 \mathrm{~A}}=0.03 \mathrm{~m}\right)$.

The front face of barrier $2 \mathrm{~B}$ is inclined at $90^{\circ}$ and has a height of $0.19 \mathrm{~m}$.

The incident divergent spherical wave resulting from detonation of the gas load is reflected on the front face of barrier 2B. The surface is inclined at $90^{\circ}$, and the angle of incidence between the wave and wall varies from $0^{\circ}$ to $70^{\circ}$ along this surface. This variation of the angle of incidence leads to an evolution of the reflection mode, with a changeover from reg- ular reflection towards Mach reflection. All configurations of barrier 2B lead to the formation of a Mach stem on the front face of the structure near the top. In the case of a regular reflection, a wave is formed on the surface and is propagated in the opposite direction to the incident wave (thus, in the direction of the blast load). This reflected wave results from the reflection of the incident wave on the barrier and interacts with the interface between the air and the detonation products shortly after the end of the detonation. Resulting overpressures are higher in the case of barriers with a vertical face with respect to the explosion than in the case of barriers with an inclined face of $45^{\circ}$.

Figure 10 shows the evolution of the maximum reflected overpressure for the various configurations of barriers $1 \mathrm{~B}$ and 2B.

\subsection{Relaxation on the top of barriers $1 B$ and $2 B$}

The Mach stem resulting from reflection of the shock wave on the front face of the barrier undergoes a relaxation at the top of the wall. The angle of deviation of this first relaxation varies according to the inclination angle of the front face of the barrier: $45^{\circ}$ for barrier $1 \mathrm{~B}$ and $90^{\circ}$ for barrier $2 \mathrm{~B}$.

The phenomenology on the top of barrier $1 \mathrm{~B}$ is identical to that for barriers $1 \mathrm{~A}$ and $2 \mathrm{~A}$. The level of overpressure decreases rapidly at the foot of the wall because of relaxation induced by the change of slope at the top. The geometry of barrier $1 \mathrm{~B}$ is different from the barriers of series 1 because of the greater thickness at the top $(e=H)$, thus enhancing the attenuation per distance covered on this surface.

The upstream face of barrier 2B has an angle of inclination of $90^{\circ}\left(\alpha_{1}=90^{\circ}\right)$. This leads to an increase in the angle 
of deviation of the first relaxation of the Mach stem on the top of the barrier. The increase in the angle of deviation thus increases the attenuation of the maximum overpressure during relaxation. The maximum overpressure then decreases more rapidly than in the case of barrier $1 \mathrm{~B}$, which has a front face inclined at $45^{\circ}$.

\subsection{Relaxation on the downstream face of barriers 1B and $2 B$}

The shock wave propagated over the top of the barrier undergoes a second relaxation during its passage over the downstream face of the barrier: "relaxation in two stages" (non-zero thickness at the top, $e \neq 0 \mathrm{~m}$ ).

For barrier $2 \mathrm{~B}$, the rear face is inclined at $45^{\circ}\left(\theta=45^{\circ}\right)$; thus, the slope angle at the top of the rear face is less than that for barrier 1B $\left(\theta=90^{\circ}\right)$. On the rear face of the barrier, the maximum overpressure undergoes less attenuation compared to barrier 1B (Fig. 10).

\section{Protective effect of barriers A and B}

\subsection{Normative distance}

The attenuation factor allows us to evaluate the protective effect of the barrier compared to a configuration-free field (without structure), as shown in equation (11):

$A_{\mathrm{P}}=\frac{\Delta P_{\mathrm{r}}^{+}}{\Delta P_{\mathrm{i}}^{+}}$

where $\Delta P_{\mathrm{i}}^{+}$is the maximum incident overpressure in the free field [6] and $\Delta P_{\mathrm{r}}^{+}$is the maximum overpressure in the presence of the protection barrier. Thus, if $A_{\mathrm{P}}$ tends towards zero, then the maximum protective effect is characterized by a new scaled distance $R_{\text {barrier }}\left[\mathrm{m} \mathrm{MJ}^{-1 / 3}\right.$ ] defined as follows:

$R_{\mathrm{barrier}}=\frac{R}{\left[E\left(1-\frac{d}{\sqrt{d^{2}+S}}\right)\right]^{1 / 3}}$,

where $R$ is the distance between the centre of the gas load and the measurement point [m], $E$ the energy released by the gas load [MJ], $d$ the distance between the centre of the charge and the lower point on the front face of the barrier [m] and $S$ the cross section $\left[\mathrm{m}^{2}\right]$. This new parameter corresponds to a normative distance which offers the major advantage of considering the form of the barrier rather than the classical parameter $n$ defined by the ratio of the ground distance behind the barrier to the barrier height [4].

The energy released by the propane-oxygen reaction is obtained by multiplying the energy per unit volume $E_{\mathrm{v}}$ by the volume $V$ of the spherical charge: $E=E_{\mathrm{V}} \times V$. By considering the density $\rho$ of the gas mixture, the relationship between $R_{\text {barrier }}$ and $Z$ (4) can be derived as follows:

$R_{\text {barrier }}=\mathrm{Z}\left(\frac{\rho}{E_{\mathrm{V}}}\right)^{1 / 3} \frac{1}{\left(1-\frac{d}{\sqrt{d^{2}+S}}\right)^{1 / 3}}$

Nevertheless, the normative distance presented here is not appropriate for a wall that is infinitely high and infinitely thin.

\subsection{Attenuation factor}

The wave that passes over the top of the protective barrier is reflected on the ground downstream from the barrier (Fig. 11). This physical phenomenon leads to an increase in the maximum overpressure downstream from the barrier.

Figure 11 shows the evolution of the attenuation factor for the four analysed geometries (barriers 1A, 2A, 1B and 2B) and for the two studied loads $\left(R_{1}\right.$ and $\left.R_{2}\right)$.

Figure 11 shows the following:

- The expression of $R_{\text {barrier }}$ allows us to highlight the effects of the type of barrier and the charge volume. Hence, we obtain four groups of curves, for different values of studied load R0 and distance $d$ : two of the groups correspond to barrier A, while the other two correspond to barrier B.

- In the case of barrier A, the attenuation may become greater than 1 if the distance $d$ tends toward $0.14 \mathrm{~m}$. Consequently, these configurations lead to the opposite effect than that expected. This situation is never present in the case of barrier B.

- The Mach stem resulting from the reflection phenomenon on the front face of the barrier B relaxes at the top of the barrier at an angle of $45^{\circ}$ for barrier $1 \mathrm{~B}$ and $90^{\circ}$ for barrier 2B. The Mach stem relaxes again on the face downstream of the barrier at two different angles: at $90^{\circ}$ and $45^{\circ}$ for barriers $1 \mathrm{~B}$ and $2 \mathrm{~B}$, respectively. This dimensioning also assigns the angle of incidence to ground level downstream from the structure: barrier $1 \mathrm{~B}, \beta_{1 \mathrm{~B}}=0^{\circ}$ and barrier $2 \mathrm{~B}, \beta_{2 \mathrm{~B}}=45^{\circ}$.

- The slope of the walls must be dimensioned according to the size of the protection zone; for example, due to the relaxation phenomenon, $\alpha_{2}$ contributes to a slight attenuation, as well as a possible reflected overpressure on the ground (possible formation of a Mach stem, $\beta>40^{\circ}$ ) and a less marked protective effect over a larger proximal but visible field (and conversely for barrier 1B).

- Thus, at a given energy $E$ regardless of the distance $d$, it is clear that barrier $B$ leads to a better protection. The result- 
Fig. 11 Evolution of the attenuation factor downstream from barriers A and B for the two studied gas loads and at various distances $d$ between the centre of the explosive charge and the foot of the barrier

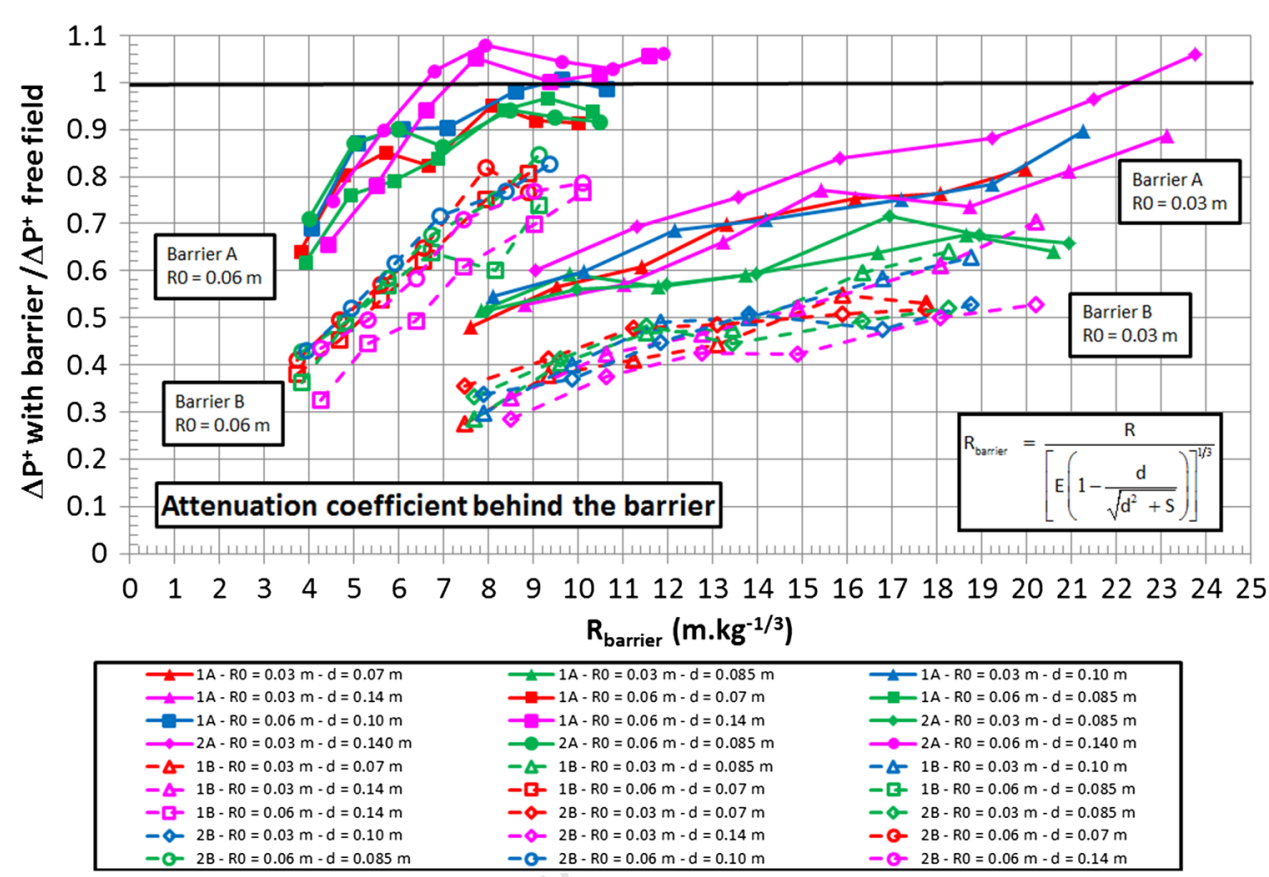

Fig. 12 Evolution of the attenuation factor downstream from barrier used by Allain [2] for different masses of TNT and distances from the charge to the foot of the barrier

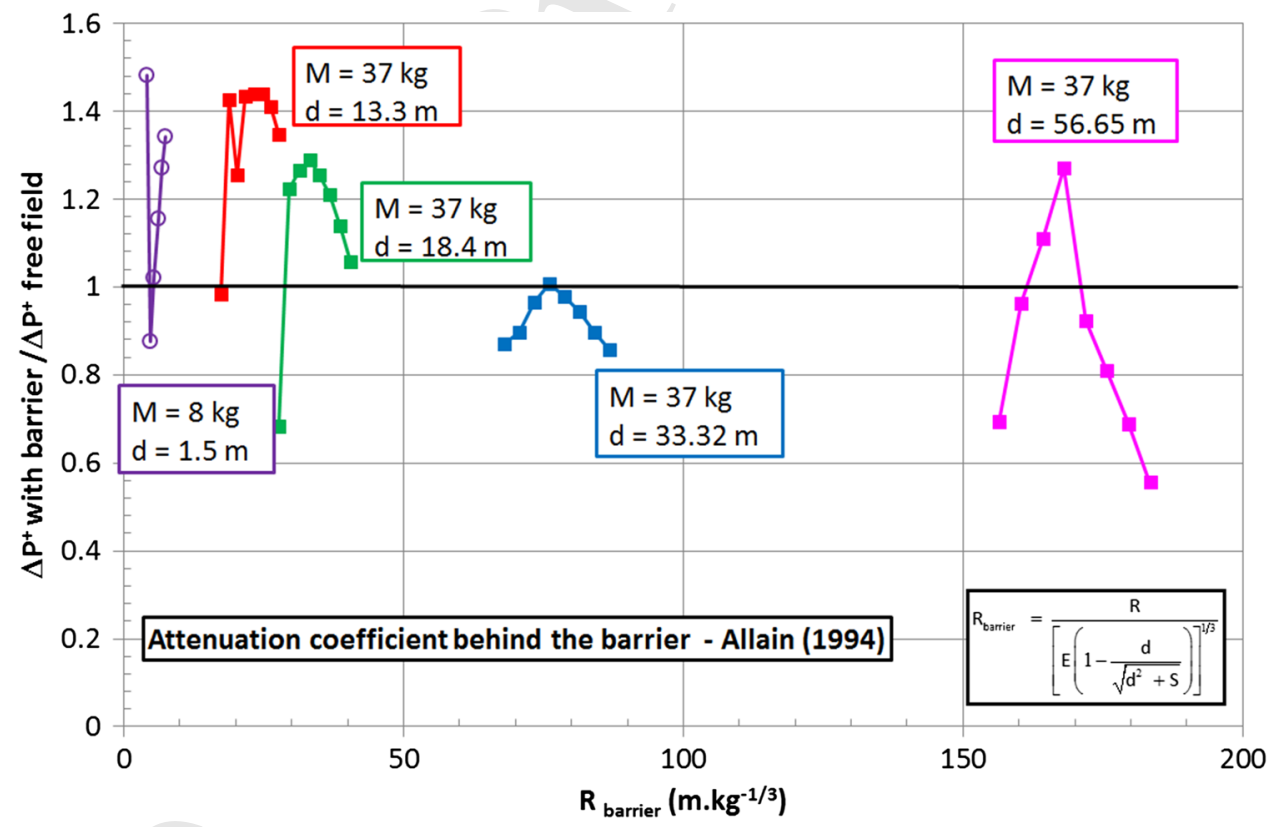

ing protective effect is nearly identical between barriers $1 \mathrm{~A}$ and $2 \mathrm{~A}$ or $1 \mathrm{~B}$ and $2 \mathrm{~B}$. The presence of a $90^{\circ}$ angle on a barrier (downstream or upstream) causes a sensitive attenuation of the overpressure compared to barriers with two angles at $45^{\circ}$. The result is confirmed for the two investigated loads $\left(R_{1}=0.03 \mathrm{~m}\right.$ and $\left.R_{2}=0.06 \mathrm{~m}\right)$. Barrier B allows an increase in the attenuation of the maximum overpressure due to a marked relaxation phenomenon (increase in the angle of deviation for one of the two relaxations, $\theta=90^{\circ}$ ). These differences can explain the variation in the attenuation coefficient between the two barrier geometries A and B (Fig. 11). The barrier geometries tested in series A thus offer less protection than those tested in series B.

\subsection{Comparison at medium scale}

- The presence of some thickness at the top of the barrier $(e \neq 0)$ allows relaxation "in two stages", with a Mach stem resulting from reflection on the front face. This recommendation of the NATO report represents 
"good practice" for the dimensioning of protective barriers $(e>0.5 \mathrm{~m})$.

- We highlight that barrier A is less effective than barrier B. To corroborate this observation obtained with smallscale experiments, the experimental results of Allain [2] are expressed versus the normative distance on Fig. 12. The barriers considered by Allain had two inclined slopes of $45^{\circ}$ without any thickness at the top and a height of $1.5 \mathrm{~m}$, using two types of TNT charges ( 8 and $37 \mathrm{~kg}$ ).

- The experimental results of Allain [2] reported here highlight the amplifying effect of "overpressure" due to the barrier. This phenomenon can be explained by the hypothesis that maintenance of the Mach number of the shock front (weak attenuation of overpressure) is caused by diffraction and relaxation on the rear face of the Mach stem (resulting from reflection on the front face) or affecting the incident wave. In turn, this imposes an angle of incidence close to $45^{\circ}$ that can approach the requirements for the formation of a new Mach stem at the end of the rear face of the barrier for certain configurations. This possible relaxation is accompanied by a reflection on the ground, possibly leading to the creation of a stronger Mach stem on overpressure.

- Consequently, the geometry of the barrier used by Allain [2] appears to be unsuitable for the protection of people, equipment and structures, thus supporting our results obtained at small scale with barrier A.

\section{Conclusion and recommendations for dimensioning}

The study of various protective barrier configurations leads to an analysis of the interaction of shock waves with barriers according to their geometrical parameters and an assessment of their impact on the protective effect.

The ideal protective barrier is a parallelepiped with significant height and thickness. Indeed, this geometry allows enhancement of the attenuation of the maximum overpressure by increasing the distance covered (Taylor waves) and favouring the presence of "strong" relaxations (angles of deviation $(\theta)$ close to $90^{\circ}$ ). Nevertheless, according to the additional constraints of dimensioning (such as limited space and financing), this type of geometry may be difficult to implement and can be "oversized" compared to the needs of the user $\left(\Delta P^{+}\right.$downstream $\ll 0.020 \mathrm{bar}$, threshold of the last affected zone $\left(Z_{5}\right)$ ). The optimal dimensioning of a protective barrier thus depends on the available resources and dimensions of the configuration of interest (position of the zone to be protected with respect to the blast load).
Thus, the user should optimize the dimensioning of the barrier based on three sets of geometrical parameters: height $(H)$ - thickness $(e)$, inclination angles of the front and rear faces $\left(\alpha_{1}\right.$ and $\left.\alpha_{2}\right)$, as well as the positioning of the barrier with respect to the load $(d)$.

The recommendations of NATO [4] appear robust and useful for promoting "good practices" in the dimensioning of protective barriers. These recommendations allow consideration of a minimal height and thickness to ensure a protective effect downstream from the barrier. Nevertheless, the choice of maximum possible height and thickness according to the available resources can be used to enhance the phenomenon of attenuation by increasing the distance covered by the shock wave over the structure. In addition, the tests conducted by Allain [2] clearly show the limited effect of this type of barrier geometry, as indicated by the small-scale experiments (barrier A).

The choice of the inclination angles of the front and rear faces also depends on the means available. Indeed, an inclination angle of $90^{\circ}$ should be used to enhance attenuation of the maximum overpressure caused by the presence of strong relaxations on the edges of the barrier. Moreover, the use of a vertical barrier face prevents the rapid formation of a Mach stem upstream (front face) and downstream from the barrier. Formation of a Mach stem leads to a recompression of the shock wave, thus reducing the protective effect of the barrier. The experimental results obtained in this study clearly demonstrate that barrier B with an inclination angle of $90^{\circ}$ is more efficient in terms of overpressure attenuation than barrier A.

However, this type of dimensioning $\left(\alpha_{1}=\alpha_{2}=90^{\circ}\right)$ also implies major constraints affecting the resistance of the structure, with the risk of projection of new fragments from the barrier (maximum considered overpressure on the front and rear faces of the barrier).

The positioning of the protective barrier relative to the explosive charge depends on the geometry of the selected barrier and the position or size of the downstream zone to be protected. Indeed, according to the slope of the wall, the flow mode can be modified by the formation of a Mach stem upstream and downstream from the protective barrier.

- If the angle of inclination is high $\left(\alpha_{1}\right.$ near to $\left.90^{\circ}\right)$, a protective barrier placed in the field close to the blast load offers a strong protective effect downstream from the barrier (important screen effect [3]. Nevertheless, this dimensioning also implies high overpressure on the upstream barrier face, in particular by deformation of the reflection due to the presence of an interface between air and detonation products in a field close to the wall and shock wave (for a gas load (stoichiometric propane-oxygen combustion), $d<0.58 \mathrm{~m} / \mathrm{MJ}^{1 / 3}$; for a condensed chemical charge (TNT), $d<0.88 \mathrm{~m} / \mathrm{kg}^{1 / 3}$ ). 
- If an angle of inclination of $90^{\circ}$ for the front face cannot be considered on an industrial site, this can be offset by using an angle of $90^{\circ}$ on the rear face since similar attenuations are obtained (comparison of barriers $1 \mathrm{~B}$ and 2B).

- If the angle of inclination of the front face is less than $90^{\circ}\left(\alpha_{1} \ll 90^{\circ}\right)$, the protective effect is also more pronounced in terms of amplitude for a barrier placed in the field close to the load (important screen effect). The overpressure reflected on the upstream face is also less marked. However, a barrier placed in the far field of the blast load offers a less important protective effect in terms of amplitude compared with a barrier placed in the near field [3].

These "good practices" can be used to guide engineers in the optimal dimensioning of protective barriers according to the configuration on the ground and the resources available ([10]). The construction of nomograms will supplement these recommendations and allow a precise evaluation of the protective effects according to the geometrical parameters of the barriers $\left(d, H, e, \alpha_{1}\right.$ and $\left.\alpha_{2}\right)$.

Acknowledgments This study is part of the BARPPRO ANR research programme (dimensioning physical protective barriers against propagation of pressure waves following an explosion). The work was carried out at the ANR and CEA, who provided financial support.

\section{References}

1. Zhou, Z.Q., Hao, H.: Prediction of airblast loads on structures behind a protective barrier. Int. J. Impact Eng. 35, 363-375 (2008)

2. Allain, L.: Barricade Influence on Blast Wave Propagation. Report, SNPE / Division Défense Espace, Vert-le-Petit, France (1994)

3. Borgers, J.: Blast walls reviewed. In: 21st International Symposium on Military Aspects of Blast and Shock, Jerusalem, Israel (2010)

4. Guide SFEPA n ${ }^{\circ}$. Guide des Bonnes Pratiques en PyrotechnieEdition Syndicat des Fabricants d'Explosifs, de Pyrotechnie et d'Artifices (2009)

5. Éveillard, S., Lardjane, N., Vinçont, J.Y., Sochet, I.: Towards a fastrunning method for blast-wave mitigation by a prismatic blast wall. Compte Rendus Mecanique 341, 625-635 (2013)

6. Sochet, I., Sauvan, P.E., Boulanger, R., Nozeres, F.: Effect of a gas charge explosion at the closed end of a gas storage system. J. Loss Prev. Process Ind. 27, 42-48 (2014)

7. Trélat, S., Sochet, I., Autrusson, B., Cheval, K., Loiseau, O.: Strong explosion near a parallelepipedic structure. Shock Waves 16, 349357 (2007)

8. Kinney, G.F., Graham, J.K.: Explosive Shocks in Air, 2nd edn. Springer-Verlag, Berlin (1985)

9. TM5-1300, U.S. Department of the Army.: Structures to Resist the Effects of Accidental Explosions. Army TM 5-1300, Navy NAVFAC P-397, AFR 88-22. Departments of the Army, Navy and Air Force, Washington, DC (2008)

10. Eveillard, S.: Propagation d'une onde de choc en présence d'une barrière de protection-PhD Orleans University, France (2013) 